\title{
NÍVEIS DE ATIVIDADE FÍSICA: UM ESTUDO COMPARATIVO ENTRE ADOLESCENTES SURDOS E OUVINTES
}

\author{
PHYSICAL ACTIVITY LEVELS: A COMPARATIVE STUDY BETWEEN DEAF AND HEARING ADOLESCENTS \\ NIVELES DEACTIVIDAD FISICA: UN ESTUDIO COMPARATIVO ENTRE ADOLESCENTES SORDOS Y OYENTES
}

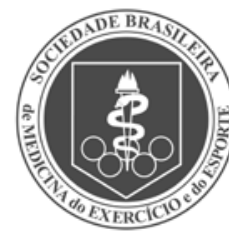

Artigo Original

Original Article

Artículo Original
Luana Foroni Andrade ${ }^{1}$

(Terapeuta educacional)

Shamyr Sulyvan de Castro

(Professor Adjunto)

1. Universidade Federal do

Triângulo Mineiro (UFTM), Programa de Pós-Graduação em Atenção à

Saúde, Uberaba, MG, Brasil.

2. Universidade Federal do

Ceará (UFC), Departamento de

Fisioterapia, Fortaleza, CE, Brasil.

\section{Correspondência:}

Programa de Pós-Graduação em

Atenção à Saúde, Universidade

Federal do Triângulo Mineiro

(UFTM). Av. Getúlio Guaritá, 107.

Abadia, Uberaba-MG, Brazil.

38025-440. luanaforoni@gmail.com

\section{RESUMO}

Introdução: A Organização Mundial da Saúde tem alertado para o crescente quadro de sedentarismo e suas consequências entre as populações. A inatividade física passou a ser o quarto principal fator de risco de saúde, tornando-se um dos maiores problemas de saúde pública na atualidade. Populações como adolescentes surdos brasileiros têm sido negligenciadas com ausência de inquéritos de saúde voltados à investigação dessa variável e proposições de estratégias que garantam equiparação de oportunidades. Objetivo: Assim, este estudo teve por objetivo verificar o nível de atividade física entre adolescentes surdos comparados a adolescentes ouvintes. Métodos: O grupo de adolescentes ouvintes mostrou-se significativamente mais ativo que os adolescentes surdos $(61,29 \%$ para $29,03 \%$ com $p<0,05)$. Resultados: O resultado da presente pesquisa evidencia que há necessidade de investigações sobre as realidades em que se desenvolvem os programas e ações voltados ao esporte para adolescentes surdos. Conclusões: imprescindível buscar as causas e propor soluções junto a órgãos gestores e secretarias ligadas ao esporte, saúde e educação para a diminuição do índice encontrado, proporcionando equiparação de oportunidades entre surdos e ouvintes.

Descritores: surdez; adolescente; exercício.

\section{ABSTRACT}

Introduction: The World Health Organization has warned about the growing condition of sedentariness and its consequences among populations. Physical inactivity has become the fourth major health risk factor, making it one of the largest public health problems today. Populations as Brazilian deaf adolescents have been neglected due to the absence of health surveys focused on the research of this variable and propositions strategies that ensure equal opportunities. Objective: This study aimed to verify the level of physical activity among deaf adolescents compared to hearing adolescents. Methods: The group of hearing adolescents was significantly more active than deaf adolescents (61.29\% to 29.03\% with $p<0.05)$. Results: The result of this research shows that there is a need for investigations on the realities in which sport programs and actions for deaf adolescents are developed. Conclusion: It is essential to seek the causes and propose solutions with the management bodies and agencies related to sports, health and education to reduce the index found, providing equal opportunities for deaf and hearing people.

Keywords: deafness; adolescent; exercise.

\section{RESUMEN}

Introducción: La Organización Mundial de la Salud ha alertado sobre el creciente cuadro de sedentarismo y sus consecuencias entre las poblaciones. La inactividad física pasó a ser el cuarto principal factor de riesgo de salud, convirtiéndose en uno de los mayores problemas de salud pública en la actualidad. Poblaciones como adolescentes sordos brasileños han sido descuidadas con ausencia de encuestas de salud dirigidas a la investigación de esa variable y proposiciones de estrategias que garanticen equiparación de oportunidades. Objetivo: Así, este estudio tuvo por objetivo verificar el nivel de actividad física entre adolescentes sordos comparados a adolescentes oyentes. Métodos: El grupo de adolescentes oyentes fue significativamente más activo que los adolescentes sordos (61,29\% para 29,03\% con $p<0,05)$. Resultados: El resultado de la presente investigación evidencia que hay necesidad de investigaciones sobre las realidades en que se desarrollan los programas y acciones dirigidos al deporte para adolescentes sordos. Conclusiones: Es imprescindible buscar las causas y proponer soluciones junto a órganos gestores y secretarías ligadas al deporte, salud y educación para la disminución del índice encontrado, proporcionando equiparación de oportunidades entre sordos y oyentes.

Descriptores: sordera; adolescente; ejercicio. 


\section{INTRODUÇÃO}

A prática de atividade física regular durante a infância e a adolescência tem sido apontada em diversos estudos como uma alternativa para diminuir as chances de desenvolvimento de doenças crônico-degenerativas na vida adulta ${ }^{1,2}$. Alternativa essa que é destacada como o primeiro passo para reverter o crescente quadro de sedentarismo e suas consequências entre as populações ${ }^{3-5}$. A Organização Mundial de Saúde ${ }^{6}$ traz que a inatividade física passou a ser o quarto principal fator de risco à saúde, segundo Seabra ${ }^{7}$, um dos maiores problemas de Saúde Pública.

Em qualquer população e cultura, a prática de atividade física tornou-se então alvo de estudos e de estratégias em cooperação de órgãos gestores e comunidade. Todavia, há escassez de pesquisas voltadas à investigação e descrição de saúde e seus estados em algumas comunidades e populações. É o caso da população de adolescentes surdos brasileiros. Inquéritos em saúde respeitando as especificidades culturais dessa comunidade têm sido escassos e até inexistentes em vários países. Segundo McKee et al. ${ }^{8}$ isso ocorre, pois essa população historicamente, devido à comunicação e às barreiras linguísticas, aparece marginalizada e excluída.

O Brasil tem apresentado ações priorizando a preocupação com o envelhecimento da população de modo ativo e saudável, a coexistência de enfermidades transmissíveis e não transmissíveis; e a consciência de que fatores sociais são determinantes à saúde 9 . Essas preocupações e iniciativas foram essenciais na elaboração e execução do presente estudo que teve por objetivo verificar o nível de atividade física entre adolescentes surdos, população em minoria linguística-cultural pouco investigada, em comparação a adolescentes ouvintes. Indo ao encontro da Política Nacional de Promoção da Saúde - PNPS ${ }^{10}$ que em seu eixo práticas corporais/atividade física propõe resgatar a prática de atividades físicas e corporais em espaços públicos, estimulando ações já existentes e consolidando pesquisas de saúde em escolares como forma de monitoramento dessas práticas.

\section{MÉTODOS}

Este estudo caracteriza-se por uma pesquisa quantitativa de natureza investigativa analítica e comparativa. Para alcançar o objetivo proposto, foram levantadas as escolas que acolhiam e trabalhavam com o maior número de alunos surdos da cidade e região, sendo a primeira uma escola filantrópica com pedagogia bilíngue e ensino com primeira língua em LIBRAS e a segunda escola regular estadual com pedagogia inclusiva. Devido à baixa frequência de sujeitos, a amostra deste estudo se caracteriza como não probabilística por conveniência. Os critérios de inclusão para participação no estudo foram: adolescentes surdos que utilizam a LS como primeira língua, na faixa etária entre 10 a 19 anos, que cursavam o ensino fundamental ou médio, sem deficiências e/ou desordens associadas à surdez.

Um segundo grupo, formado por adolescentes ouvintes com características similares (gênero e idade), foi selecionado com o objetivo de comparar as informações coletadas no primeiro grupo. Os critérios de inclusão para este segundo grupo seguiam os mesmos critérios do primeiro com exceção do domínio da língua de sinais. Assim, cada grupo foi composto por 31 adolescentes. Todos os participantes assinaram o termo de consentimento livre e esclarecido.

Para coleta dos dados foi utilizado o instrumento Questionário de Atividade Física para Adolescentes (QAFA) validado através dos estudos de Florindo e colaboradores ${ }^{11}$, sendo sua validade e reprodutibilidade em adolescentes brasileiros confirmadas em estudos posteriores ${ }^{12-14}$. Através da ficha do aluno, cedida pelas escolas participantes foram coletados ainda dados como: idade, gênero, escolaridade, surdez ou ausência desta.
O QAFA abrange as atividades físicas ligadas ao esporte em variados espaços e a atividade de locomoção da casa até a escola, calculando em minutos o tempo gasto nessas atividades em proporções diferentes (dia/semana/mês/ano). No presente estudo o gasto foi calculado em minutos por semana com ponto de corte de trezentos minutos semanais seguindo diretrizes de estudos e órgãos internacionais importantes que são referências aos estudos nacionais ${ }^{15,16}$. Gera-se ao final uma variável dicotômica, insuficientemente ativo ou ativo, para classificação.

Para garantir equiparação de oportunidades a ambos os grupos o instrumento e os documentos envolvidos na pesquisa foram traduzidos em LIBRAS. A tradução e adaptação transcultural do instrumento seguiu as orientações encontradas nos estudos de Borsa et al. ${ }^{17}$. Os encontros entre os pesquisadores, tradutores/intérpretes de LIBRAS e membros da comunidade foram realizados buscando equivalência semântica, equivalência idiomática, equivalência experiencial e equivalência conceitual.

Para análise dos dados utilizou-se análise estatística exploratória, sendo na fase descritiva utilizados números brutos, frequências simples e porcentagens e na análise inferencial foi utilizada comparação de dados a partir dos grupos, surdos e ouvintes. As variáveis contínuas foram expressas por medidas de tendência central e dispersão e as categóricas por frequência através do teste de Qui-quadrado com $\mathrm{p}<0,05$.

A presente pesquisa obteve aprovação através do Comitê de Ética e Pesquisa da Universidade Federal do Triângulo Mineiro (CEP/UFTM) a partir do protocolo 2570.

\section{RESULTADOS}

Os resultados das informações coletadas nas duas amostras, adolescentes surdos e ouvintes, foram descritos em tabelas. A Tabela 1 apresenta a descrição dos participantes segundo a escola de origem, gênero e idade.

A variável escolaridade apresentou um número maior de adolescentes surdos cursando o ensino fundamental $67,74 \%$, enquanto apenas $32,67 \%$ dos adolescentes ouvintes cursavam esse mesmo nível de ensino. Os alunos ouvintes apresentaram porcentagem de 67,33\% cursando o ensino médio sendo que apenas 32,26\% dos adolescentes surdos cursam esse nível. As médias de idade dos dois grupos foram $14,87( \pm 3,26)$ anos para os adolescentes surdos e de 15,38 $( \pm 1,90)$ anos para os adolescentes ouvintes.

Os NAFs foram descritos na Tabela 2 a partir do teste de Qui-quadrado para comparação das frequências nos grupos. Os resultados apontam que $25,81 \%$ dos alunos surdos e 61,29\% de alunos ouvintes foram classificados como ativos em relação ao NAF relacionado ao esporte. O NAF total, relacionado à locomoção e ao esporte, alcançou a expressividade de apenas 29,03\% de sujeitos ativos no grupo de surdos, e de 61,29\% de sujeitos ativos no grupo de adolescentes ouvintes $(p<0,05)$.

Tabela 1. Descrição da amostra segundo as variáveis escola, sexo e escolaridade (2014, Uberaba-MG).

\begin{tabular}{c|c|c|c|c|c|c}
\hline Variáveis & \multicolumn{2}{|c|}{ Surdos } & \multicolumn{2}{c|}{ Ouvintes } & \multicolumn{2}{c}{ Total } \\
\hline Escola & $\mathbf{n ( 3 1 )}$ & $\mathbf{( \% )} \mathbf{1 0 0}$ & $\mathbf{n}(\mathbf{3 1})$ & $\mathbf{( \% )} \mathbf{1 0 0}$ & $\mathbf{n}(\mathbf{6 2})$ & $\mathbf{( \% )} \mathbf{1 0 0}$ \\
\hline Bilíngue - Libras/Português & 19 & 61,29 & 0 & 0 & 19 & 30,65 \\
\hline Escola Regular & 12 & 38,71 & 31 & 100 & 43 & 69,35 \\
\hline Sexo & & & & & & \\
\hline Feminino & 13 & 41,94 & 13 & 41,94 & 26 & 41,94 \\
\hline Masculino & 18 & 58,06 & 18 & 58,06 & 36 & 58,06 \\
\hline Escolaridade & & & & & & \\
\hline Ensino Fundamental & 21 & 67,74 & 10 & 32,67 & 31 & 50 \\
\hline Ensino Médio & 10 & 32,26 & 21 & 67,33 & 31 & 50 \\
\hline Eonte: Elaborado pelos autores,2015. & \multicolumn{7}{|c|}{}
\end{tabular}


Tabela 2. Nível de Atividade Física (NAF) a partir da comparação dos grupos (2014, Uberaba-MG).

\begin{tabular}{c|c|c|c|c|c|c|c}
\hline Variáveis & \multicolumn{2}{|c|}{ Surdos } & \multicolumn{2}{c|}{ Ouvintes } & \multicolumn{2}{c|}{ Total } & \\
\hline & $\mathbf{n ( 3 1 )}$ & $\mathbf{( \% )} \mathbf{1 0 0}$ & $\mathbf{n}(\mathbf{3 1})$ & $\mathbf{( \% )} \mathbf{1 0 0}$ & $\mathbf{n}(\mathbf{6 2})$ & $\mathbf{( \% )} \mathbf{1 0 0}$ & $\mathbf{p}$ \\
\hline NAF relacionada ao esporte & & & & & & & \\
\hline Ativo & 8 & 25,81 & 19 & 61,29 & 27 & 43,55 & \multirow{2}{*}{0,005} \\
\hline Insuficientemente ativo & 23 & 74,19 & 12 & 38,71 & 35 & 56,45 & \\
\hline NAF total & & & & & & & \\
\hline Ativo & 9 & 29,03 & 19 & 61,29 & 28 & 45,16 & \multirow{2}{*}{0,011} \\
\hline Insuficientemente ativo & 22 & 70,97 & 12 & 38,71 & 34 & 54,84 & \\
\hline *teste de Qui-quadrado. Fonte: Elaborado pelos autores, 2015.
\end{tabular}

\section{DISCUSSÃO}

As dificuldades encontradas no ensino da língua oral aos surdos e na promoção do uso e domínio da língua de sinais na comunidade em geral têm impactos profundos nas diversas áreas de ocupação dessa população. Nos estudos de Marin e Góes ${ }^{18}$ discussões com enfoque nas consequências nas atividades cotidianas são levantadas ressaltando a necessidade da companhia de um ouvinte para estabelecer comunicação efetiva em diversos espaços e em diferentes momentos. As atividades ocupacionais que envolvem atividades de lazer e atividades esportivas não são diferentes quando o direito ao atendimento, acoIhimento e oferecimento de informações, recursos e treinamentos de acordo com sua necessidade, uso da língua de sinais, é violado. Direitos esses garantidos em leis e documentos como os decretos 5.626/2005 e 10.436/2002, o manual" A Pessoa com Deficiência e o Sistema Único de Saúde"e a Convenção sobre os Direitos das Pessoas com Deficiência ${ }^{19,20}$.

Neste estudo a população de adolescentes surdos foi apontada como mais insuficientemente ativa que a população de adolescentes ouvintes. Estudos com a população de adolescentes surdos brasileiros, especificamente, são escassos. Todavia, Interdonato e Greguol ${ }^{21}$ em uma pesquisa com a população de adolescentes com deficiência auditiva encontroram que cerca de 60\% da amostra também era irregularmente ativa, o que corresponde a insuficientemente ativo para a classificação utilizada pelo instrumento QAFA. Embora o estudo tenha sido realizado com deficientes auditivos e não com a população surda propriamente, que se diferencia do primeiro grupo através de elementos culturais próprios, os resultados colaboram com a discussão e apontam a necessidade de investir em estratégias de estímulo à atividade física com a população que possuem perda auditiva.

Teixeira et al. ${ }^{22}$ destacam que a deficiência auditiva em si não implica em restrições significativas em relação a prática de atividade física ou de esportes. Fatores como falta de informação ou boa vontade, diminuindo a oportunidade de vivências motoras dessa população podem ter implicações bem mais significativas. A vivência motora e seu desempenho empobrecido na infância podem ter impacto especialmente negativo na participação futura nas atividades esportivas. Consequentemente crianças surdas podem apresentar estilos de vida menos ativos durante a adolescência e vida adulta que seus pares ouvintes quando apresentam esse desempenho motor diminuído ${ }^{23,24}$.

Os autores Ellis et al..$^{25}$, em um estudo mais recente, encontraram em uma amostra de 128 crianças surdas evidências de que as crianças surdas podem apresentar níveis adequados de aptidão física quando são realizadas simples modificações na administração de testes levando em conta principalmente a comunicação e compreensão correta das informações. Este estudo ressalta a importância de adequação dos instrumentos e estratégias à necessidade da população investigada.

Os espaços que oferecem programas e intervenções voltadas à atividade física regular, especificamente de esportes, por vezes apresentam estrutura física e humana pouco acessível e capacitada para suprir as necessidades dessa população. O que apresenta um violação de direitos já garantido em importantes documentos e órgãos como nos apresenta a Secretaria de Direitos Humanos ${ }^{26}$ que discute nas Políticas Públicas de Esporte Cultura, Lazer e Turismo a preocupação com a universalização das estruturas físicas nas ações paradesportivas com contratação, capacitação e formação continuada de profissional especializado; exibição das surdolimpíadas com legenda em LIBRAS; e direito à prática de educação física escolar e participação dessa população em eventos esportivos nas esferas municipais, estaduais e federal.

O resultado da presente pesquisa, evidenciando baixa frequência de adolescentes surdos ativos no grupo estudado também traz para discussão a necessidade de investigações que descrevam as realidades em que se desenvolvem os programas e ações voltados ao esporte para adolescentes surdos brasileiros. Pois, pensando em ambientes em diversidades a inclusão social deve ser priorizada e entendida como um processo em que a sociedade adapta seus sistemas para incluir, buscando equacionar problemas e efetivar propostas para equiparação de oportunidades ${ }^{27}$.

O termo "equiparação de oportunidades", processo em que os diversos sistemas da sociedade e do ambiente tornam-se disponíveis a todos, garantindo o direito de permanecer em comunidade recebendo o apoio e estruturas nas diversas esferas: saúde, educação, emprego e serviços sociais ${ }^{20,27}$, tornou-se mais difundindo nas últimas décadas. Todavia, pesquisas que relatem e investiguem a execução dessa proposta de maneira equitativa são escassas.

Na atualidade, os surdos brasileiros passaram a ocupar espaços sociais e acadêmicos modificando as relações interpessoais e profissionais que envolvem surdos e ouvintes. As diferenças tornaram-se mais visíveis, reconhecidas, respeitadas e valorizadas como formas humanas legítimas de existência. O que não se permite mais olhar o surdo como deficiente, mas como aquele que experiência o mundo pela visão ${ }^{28}$. Essa nova experiência e relação fazem com que questionemos o resultado da presente pesquisa como resultado não de uma condição biológica, mas uma condição cultural de sujeitos imersos em ambientes poucos sensíveis a diversidade.

Se ainda os adolescentes ouvintes apresentaram nessa pesquisa ser mais ativos que os adolescentes surdos, destaca-se a necessidade de um olhar sensível a esse resultado e um maior monitoramento também dessa população, uma vez que estudos nacionais envolvendo a população de adolescentes ouvintes têm expostos achados pouco satisfatórios em relação aos níveis de atividade física. No estudo de Hallal et al. ${ }^{29}$, por exemplo, apenas $43,1 \%$ da amostra de adolescentes do 90 ano do ensino fundamental de escolas públicas e privadas de todas as capitais brasileiras e do Distrito Federal foram classificados como ativos. Na cidade de Pelotas, em uma amostra de 4452 adolescentes, $48,7 \%$ dos meninos e $67,5 \%$ das meninas mostraram-se insuficientemente ativos ${ }^{30}$.

Os resultados em ambas as amostras são importantes para traçar metas e subsidiar o planejamento de políticas e programas primeiramente locais ou regionais para o estímulo da prática saudável de atividade física entre o público jovem. Estendendo essas estratégias para outras amostras nacionais populacionais com perfis similares. Gestores de Esporte, Saúde e Educação, podem ainda ser beneficiados com os resultados sobre os níveis de atividade física apresentados na presente pesquisa, ao estabelecerem ações de monitoramento destes escolares avançando com propostas sensíveis para equiparação de ofertas adequadas e criação de estratégias modelos com maiores facilitadores para a realização dessas práticas.

O acesso adequado ao público com surdez, respeitando o direito de igualdade e justiça inerente aos diferentes grupos populacionais, amplia consequentemente a possibilidade de avanços em índices sociais e de 
saúde para essa comunidade. Profissionais da educação e da saúde, podem através das informações levantadas no estudo, serem beneficiados devido ao pequeno volume ou até ausência de inquéritos que envolvem essa variável direcionados à população de adolescentes surdos. Vislumbrando o cenário atual e propondo novos caminhos que favoreçam a comunidade surda e a comunidade ouvinte equitativamente.

Verifica-se ao final, que este estudo possui como limitação a não investigação dos espaços e estratégias utilizados para a realização dessas atividades, bem como o levantamento da realização de equiparação de oportunidades entre as duas populações, adolescentes surdos e ouvintes. Sugere-se que novos estudos sejam realizados buscando explorar essa lacuna, somando as informações angariadas na presente pesquisa. Promovendo assim, o monitoramento dessa amostra para maior controle e investimentos de fomentos e ações para melhorias.

\section{CONCLUSÃO}

Neste estudo foram encontradas diferenças estatisticamente significativas nos NAFs entre adolescentes surdos e ouvintes. Sendo o grupo de surdos mais insuficientemente ativos que os ouvintes. Esse achado destaca a necessidade de ações sociais e acadêmicas a partir de contato com órgãos gestores e secretarias ligadas ao esporte, saúde e educação, bem como grupos de pesquisa. Essas ações devem buscar alternativas e o desenvolvimento de novos estudos no intuito de levantar as causas e propor soluções que diminuam o índice encontrado, aumentando assim a equiparação de oportunidades entre as surdos e ouvintes.

Todos os autores declararam não haver qualquer potencial conflito de interesses referente a este artigo.

CONTRIBUIÇÕES DOS AUTORES: Cada autor contribuiu individual e significativamente para o desenvolvimento do manuscrito. LFA (0000-0003-2765-1535)* e SSC (0000-0002-2661-7899)* contribuíram substancialmente na concepção do artigo, aquisição, análise e interpretação dos dados, discussão dos resultados, redação do trabalho, revisão crítica do conteúdo intelectual, revisão e aprovação final da versão do manuscrito. *ORCID (Open Researcher and Contributor ID).

\section{REFERÊNCIA}

1. Matsudo SMM, Araujo TL, Matsudo VKR, Andrade DR, Valquer W. Nível de atividade física em crianças e adolescentes de diferentes regiões de desenvolvimento. Rev Bras Ativ Fís Saúde. 1998;3(4):14-26.

2. Tassitano RM, Bezerra J, Tenório MCM, Colares V, Barros MVG, Hallal PC. Atividade física em adolescentes brasileiros: uma revisão sistemática [acesso em 2014 mar 08]. Rev Bras Cineantropom Desempenho Hum. 2007;9(1):55-60. Disponível em <http://www.luzimarteixeira.com.br/wp-content/uploads/2010/04/ atividade-fisica-em-adolescentes-brasileiros-uma-revisao-sistematica.pdf>

3. Trost SG, Owen N, Bauman AE, Sallis JF, Brown W. Correlates of adults' participation in physical activity: review and update. Med Sci Sports Exerc. 2002;34(12):1996-2001.

4. World Health Organization. Global strategy on diet, physical activity and health. WHO; 2004 [acessO em 2014 mar 8]. Disponível em <http://www.who.int/dietphysicalactivity/strategy/eb11344/strategy_english_web.pdf>

5. Junior MRA, Araújo CLP, Pereira FM. Atividades físicas e esportivas na adolescência: mudanças de preferências ao longo das últimas décadas [acesso em 2015 mar 24]. Rev Bras Educ Fís Esp. 2006;20(1):51-8 Disponível em <http://www.revistas.usp.br/rbefe/article/view/16613/18326>

6. World Health Organization. Global Health Risks: Mortality and burden of disease attributable to selected major risks [acesso em 2015 mar 24]. Geneve:WHO; 2009. Disponivel em <http://www.who.int/healthinfo/ global_burden_disease/GlobalHealthRisks_report_full.pdf>

7. Seabra AF, Mendonça DM, Thomis MA, Anjos LA, Maia JA. Determinantes biológicos e sócio-culturais associados à prática de atividade física de adolescentes. Cad Saúde Pública; 2008;24(4):721-36.

8. McKee M, Thew D, Starr M, Kushalnagar P, Reid JT, Graybill P, et al. Engaging the Deaf American sign language community: lessons from a community-based participatory research center. Prog Community Health Partnersh. 2012;6(3):321-9.

9. Brasil, Ministério da Saúde, Secretaria de Atenção em Saúde, Departamento de Açōes Programáticas Estratégicas, Área Técnica de Saúde do Adolescente e do Jovem. Diretrizes nacionais para a atenção integral à saúde de adolescentes e jovens na promoção, proteção e recuperação da saúde. 2010 [acesso em 2015 mar 20]. Disponível em: <http://bvsms.saude.gov.br/bvs/publicacoes/diretrizes_nacionais_atencao_saude_adolescentes_jovens_promocao_saude.pdf>

10. Brasil, Ministério da Saúde, Secretaria de Vigilância em Saúde, Secretaria de Atenção à Saúde. Política Nacional de Promoção da Saúde. 2a ed. 2006 [acesso em 2015 mar 20]. Disponível em: <http://bvsms. saude.gov.br/bvs/publicacoes/PoliticaNacionalPromocaoSaude.pdf>

11. Florindo AA, Romero A, Peres SV, Silva MV, Slater B. Desenvolvimento e validação de um questionário de avaliação da atividade física para adolescentes. Rev Saude Publica. 2006;40(5):802-9.

12. Ribeiro EHC, Florindo AA. efeitos de um programa de intervenção no nível de atividade física de adolescentes de escolas públicas de uma região de baixo nível socioeconômico: descrição dos métodos utilizados. Rev Bras Ativ Fís Saúde. 2010;15(1):28-34.

13. Romero A, Slater B, Florindo AA, Latorre MRD, Cezar C, Silva MV. Determinantes do índice de massa corporal em adolescentes de escolas públicas de Piracicaba, São Paulo. Ciênc Saúde Col. 2010;15(1):141-9.

14. Romero A, Florindo AA, Voci SM, Slater B. Reprodutibilidade de questionário informatizado de atividade física em adolescentes. Rev Bras Ativ Fís Saúde. 2011;16(3):234-9.
15. Pate RR, Freedson PS, Sallis JF, Taylor WC, Sirard J, Trost SG, Dowda M. Compliance with physical activity guidelines: prevalence in a population of children and youth. Ann Epidemiol. 2002;12(5):303-8.

16. World Health Organization. Global recommendations on physical activity for health: 5-17 years old [acesso em 2014 nov 18]. Geneve:WHO; 2011. Disponivel em: <http://www.who.int/dietphysicalactivity/ physical-activity-recommendations-5-17years.pdf?ua=1>

17. Borsa JC, Damásio BF, Bandeira DR. Adaptação e validação de instrumentos psicológicos entre culturas: algumas considerações. Paidéia. 2012;22(53):423-32

18. Marin CR, Góes MCR. A experiência de pessoas surdas em esferas de atividade do cotidiano. Cad Cedes 2006;26(69):231-49.

19. Brasil, Ministério da Saúde, Secretaria de Atenção à Saúde, Departamento de Ações Programáticas Estratégicas. A pessoa com deficiência e o Sistema Único de Saúde, 2007 [acesso em 2015 mar 20]. Disponível em: http://bvsms.saude.gov.br/bvs/publicacoes/07_0327_M.pdf.

20. Brasil, Secretaria Especial dos Direitos Humanos, Coordenadoria Nacional para Integração da Pessoa Portadora de Deficiência. A Convenção sobre os Direitos das Pessoas com Deficiência Comentada. 2008 [acesso em 2015 mar 24]. Disponível em: https://www.governoeletronico.gov.br/documentos-e-arquivos/A\%20Convencao\%20sobre\%20os\%20Direitos\%20das\%20Pessoas\%20com\%20Deficiencia\%20 Comentada.pdf

21. Interdonato GC, Greguol M. Qualidade de vida e prática habitual de atividade física em adolescentes com deficiência. Rev Bras Crescimento Desenvolv Hum. 2011;21(2):282-95.

22. Teixeira L, Vanícola MC, Gorgatti MG. Deficiência auditiva. In: Teixeira L. Atividade física adaptada e saúde: da teoria à prática. Barueri, SP: Phorte, 2008. p. 353-62.

23. Hartman E, Houwen S, Visscher C. Motor skill performance and sports participation in deaf elementary school children. Adapt Phys Activ Q. 2011;28(2):132-45.

24. Dummer GM, Haubenstricker JL, Stewart, DA. Motor skill performances of children who are deaf. Adap Phys Activ Q. 1996;13(4):400-14.

25. Ellis MK, Lieberman $L$, Dummer GM. Parent influences on physical activity participation and physica fitness of deaf children. J Deaf Stud Deaf Educ. 2014;19(2):270-81.

26. Brasil, Secretaria de Direitos Humanos, Secretaria de Direitos Humanos da Presidência da República SDH/ PR, Secretaria Nacional de Promoção dos Direitos da Pessoa com Deficiência SNPD. Esporte, cultura e laze [acesso em 2015 mar 24]. Disponível em: <http://www.pessoacomdeficiencia.gov.br/app/node/518>

27. Sassaki RK. Inclusão: construindo uma sociedade para todos. 8a ed. São Paulo: WVA; 2010.

28. Rodrigues $\mathrm{CH}$, Quadros RM. Diferenças e linguagens: a visibilidade dos ganhos surdos na atualidade. Rev Teias. 2015;16(40):72-88.

29. Hallal PC, Knuth AG, Cruz DKA, Mendes MI, Malta DC. Prática de atividade física em adolescentes brasileiros. Ciênc Saúde Col. 2010;15(2):3035-42

30. Gonçalves H, Hallal PC, Amorim TC, Araújo CLP, Menezes AMB. Fatores socioculturais e nível de atividade física no início da adolescência. Rev Panam Salud Publica. 2007;22(4):246-53. 